\title{
Combined Letrozole and Clomiphene versus Letrozole with low dose gonadotropin protocol for ovulation induction in infertile clomiphene-resistant women with polycystic ovary syndrome: Comparative study
}

\author{
Mervat Mohamed Harira
}

Department of Obstetrics and Gynecology, Faculty of Medicine, Zagazig University, Egypt

\begin{abstract}
Objectives: To compare the effect of combined clomiphene citrate (100mg) and Letrozole (5mg) versus Letrozole (5mg) followed by gonadotropin injection for one cycle in CC resistant PCOs patients.

Design: Prospective randomized trial.

Setting: Infertility Outpatient Clinic and Cytogenetic and Endoscopy Unit of Obstetrics and Gynecology Department of Zagazig University Hospitals.

Study group: About 212 PCOs patients with CC resistance (anovulatory response with CC at a dose of 100-150 mg for 3 cycles) were divided into two groups (106 in each group).

Intervention: A studied group was randomly divided into two groups. Group A received combined Letrozole 5mg ( 2 tablets) in the morning and clomid $100 \mathrm{mg}$ ( 2 tablets) in the evening from the $3^{\text {rd }}$ day of menses for 5 days, group B received Letrozole $5 \mathrm{mg}$ ( 2 tablets) from the $3^{\text {rd }}$ day of menses for 5 days followed by human menopausal gonadotropin (hMG) injection every other day from cycle day 7. Moreover, ultrasound folliculometry started on cycle day 10 then the dose was adjusted according to follicular size. Both groups received treatment regimen for only one cycle.

Results: There was higher ovulation rate $(85.8 \%)$ in letrozole plus hmG group than in the other group receiving letrozole and CC $(80.2 \%)$, but with no statistical significant difference $(P$-value $=0.65)$. But, there was statistical significant difference between both groups regarding stimulation characters including total number of mature follicles $(\geq 18 \mathrm{~mm}) 2.1 \pm 1.01$ in combined letrozole and CC was greater in group A than in group B $(1.66 \pm 0.98)$ with $(P-$ value $=0.009)$. Stimulation days were longer in group B $(18.2 \pm 2.5)$ than in group A $(16.2 \pm 2.1)$. Mean total E2 on the day of hCG administration was significantly higher among patients in the clomiphene-letrozole group as compared with that in the other group of letrozole with hmG ( $650 \pm 190.3$ and $540 \pm 180.5$, respectively). Also, higher day 21 serum progesterone in group A $(11 \pm 1.1)$ than $(9.5 \pm 0.9)$ in group B. But, there was no statistical significant difference in mean endometrial thickness in both groups (A and B) $(9.6 \pm 1.7$ versus $9.8 \pm 1.5 \mathrm{~mm}$, respectively) $(P$-value $=0.41)$. The pregnancy rate did not show any statistical significant difference in both groups $(P$-value $=0.45) ; 32$ patients out of 106 (30.1\%) in group A compared with 27 patients (25.4\%) in group B. Four cases were aborted in group A compared with 3 cases in group B. Four cases had twin pregnancy in group A with only 3 cases in group B. Also, there was no significant difference between both groups regarding ovarian hyperstimulation; as there were 2 cases with combined clomid and letrozole group and one case only in letrozole plus hmG group.

Conclusion: This study showed that lerozole is an effective medication when combined with CC or sequentially with $\mathrm{hmG}$ injection in improving the ovulation and pregnancy rate in CC-resistant PCOs patients with higher results when combined with hmG injection, but did not reach statistical significance.
\end{abstract}

Key Words: CC resistance, clomid, combination therapy, letrozole, polycystic ovary syndrome

Received: 22 July 2018, Accepted: 14 August 2018

Corresponding Author: Mervat Mohamed Harira, Lecturer of Obstetrics and Gynecology, Faculty of Medicine, Zagazig University, Egypt, Tel.: 01021106654,01283118201,E-mail: harira_mervat@yahoo.com

ISSN: 2090-7265, November 2018, Vol.8, No. 4

\section{INTRODUCTION}

Polycystic ovary syndrome (PCOS) is a common endocrine disorder in females of reproductive age manifested by different clinical variations with about $6.8 \%-18 \%$ prevalence in the general population. It is one of the commonest causes of anovulatory infertility, while $55 \%-70 \%$ of PCOS patients were presented with chronic anovulation ${ }^{[1,2]}$. Clomiphene citrate $(\mathrm{CC})$ is considered the traditional treatment therapy and the first treatment option used for achieving ovulation in those PCO patients. However, about $25 \%$ of them will not respond at all to $\mathrm{CC}$ and were considered to be "clomiphene resistant" ${ }^{[3]}$. 
Clomiphene citrate is an orally active nonsteroidal drug with mixed estrogen agonist/antagonist properties inducing ovarian stimulation depending on its structural similarities to estrogen, as it competes for and binds to nuclear estrogen receptors of hypothalamus leading to their depletion, thus producing a state of hypoestrogenemia that in turn triggering the negative feedback compensatory mechanisms with subsequent release of gonadotropin hormone (the desirable FSH and the undesirable LH rise) ${ }^{[4]}$.

$\mathrm{CC}$ increases $\mathrm{GnRH}$ pulse frequency when given to ovulatory patient; while in anovulatory PCOS, it increases pulse amplitude rather than frequency (those with already abnormally (high) GnRH pulse frequency $)^{[5]}$. But, there are some drawbacks with $\mathrm{CC}$ therapy ; $15-20 \%$ of women will not ovulate ${ }^{[6]}$, even those who ovulate have lower conception rate ${ }^{[7]}$. This discrepancy between ovulation rate $80 \%$ and pregnancy rate $40 \%$ may be attributed to its undesirable deleterious antiestrogenic effect on endometrial proliferation and cervical mucus quantity and quality. In addition, CC unlike estrogen remains bound to receptors for prolonged time (up to 2 weeks) ; thus interfering with receptor recycling ${ }^{[8]}$. Clomiphene resistance is defined as three cycles of failure to ovulate, but six cycles of ovulation without pregnancy is known as $\mathrm{CC}$ failure ${ }^{[9]}$.

Letrozole, a selective reversible third-generation aromatase inhibitor, has been used for ovulation induction with no deleterious effect on endometrium. It acts by inhibiting the conversion of androgens to estrogen creating an environment of hypoestrogenemia similar to the central reduction of negative feedback done by $\mathrm{CC}^{[10]}$. Letrozole may be superior or at least equal to CC in ovulation (60$80 \%$ ) and pregnancy rates in anovulatory PCOS women. Also, it induces ovulation in $62 \%$ with pregnancy rate $14.7 \%$ in patients with improper response to clomiphene or $\mathrm{CC}$ resistant ${ }^{[11]}$. In addition, Letrozole is rapidly eliminated from circulation due to shorter half-life with monofollicular growth, lower preovulatory estradiol (E2) level with thicker endometrium. Letrozole does not deplete estrogen receptors and has no antiestrogenic peripheral actions. Therefore, it has no detrimental effect on endometrial receptivity nor cervical mucus quality with safety on the fetus ${ }^{[12]}$.

Gonadotropins are of the treatment modalities suggested for CC resistant PCOS patients that may result in increased levels of luteinizing hormone and FSH. But, this treatment has many drawbacks, including the need for meticulous and continuous monitoring with serial hormonal and sonographic evaluation ${ }^{[4]}$, in addition to very narrow therapeutic index. Suboptimal dose of gonadotropin will not cause ovulation and small increase in the dose can potentially cause ovarian hyperstimulation syndrome in (PCOS) patients already with increased susceptibility to hyperstimulation syndrome due to the formation of multiple follicles. Multiple pregnancies are another adverse effects of these drugs with too much $\operatorname{cost}^{[4]}$. Regarding all these side effects of gonadotropins, in this study, we tried to detect the effect of minimizing their dose (Letrozole with low dose hMG regimen) or avoid their use (combined Letrozole and CC regimen) with monofollicular ovulation in clomiphene-resistant PCOS patients regarding both ovulation and pregnancy rate.

\section{PATIENTS AND METHODS}

\section{Type and place of study:}

This current study is a randomized, prospective study conducted in Cytogenetic and Endoscopy Unit and the Fertility Outpatient Clinic of Obstetrics and Gynaecology Department of Zagazig University, Egypt in the period from June 2016 to June 2017 after being approved by the local Institutional Review Board (IRB) and all participants gave their written informed consent before their inclusion in the study.

\section{Participants:}

The study included 220 anovulatory PCOS infertile women with CC resistance. Diagnosis of PCOS was based on the revised Rotterdam 2003 Criteria, in which at least two of the following three criteria were met (oligomenorrhea or amenorrhea, clinical hyperandrogenism and/or hyperandrogenemia and ultrasonographic polycystic ovaries) ${ }^{[13]}$. All patients were presented with oligoamenorrhea or anovulatory cycles for at least one year. All of the patients had normal serum PRL, TSH and 17OH-P. Those diagnosed anovulatory PCOS women included in the study were previously treated with 100-150 mg of CC daily from the 3rd day of cycle for 5 days for two to three cycles with persistent anovulation (CC resistant).

The inclusion criteria were CC-resistant PCOS (persistent anovulation for 2 to 3 cycles with 100-150 $\mathrm{mg}$ of (C), women who were $\leq 35$ years old with patent both tubes (normal hysterosalpingogram or laparoscopy), no history of pelvic surgery and/or pelvic inflammatory disease and no history of treatment by exogenous gonadotropin nor a history of laparoscopic ovarian drilling or cystectomy and normal semen analysis of her partner according to the 2010 World Health Organization Criteria within the last 6 months. Exclusion criteria were the presence of any other infertility factors rather than anovulatory PCOS, such as hypothalamic amenorrhea, uterine pathology such as leiomyoma, adenomyosis or congenital uterine malformation, hypersensitivity or contraindications to Letrozole.

\section{Sample size and Method of Randomization:}

Sample size : as pregnancy rate from previous two studies of both groups were $24.7 \%$ for Letrozole plus 
$\mathrm{hMG}^{[14]}$ and $42 \%$ for Letrozole combined with $\mathrm{CC}^{[15]}$ so with power $80 \%$ and confidence $95 \%$ sample will be 212 (106 in each group). Patients were randomly allocated using a computer-generated random sequence and the randomization list was held in a secure box and the participants were assigned to their groups using sequentially-numbered opaque sealed envelopes that were opened at the beginning of the study. These PCOS women with resistance to $\mathrm{CC}$ after 3 to 4 cycles of $\mathrm{CC}$ were randomized into two treatment groups: Group A (Letrozole and Clomid) included 107 patients who received combination therapy of clomiphene and Letrozole and Group B (Letrozole plus hmG group) included 108 patients.

In both groups, withdrawal bleeding was achieved using $10 \mathrm{mg}$ of progestogen tablets for 5 days before stimulation in those with amenorrhea. Patients of group A received $5 \mathrm{mg}$ of Letrozole oral tablets in the morning (Femara; Novartis Pharma Services, Switzerland) combined with $100 \mathrm{mg}$ of $\mathrm{CC}$ at night (Clomid; Global Napi Pharmaceuticals, Cairo, Egypt) for 5 days starting on day 3 of menstruation.

Whereas, all patients in group B received Letrozole $5 \mathrm{mg}$ (Femara; Novartis, Pharma Services, Switzerland) oral tablets in the morning from the $3^{\text {rd }}$ day of cycle for 5 days followed by highly purified $\mathrm{hmG}$ (Fostimon 75 IU), Urofollitropin (FSH) (IBSA, Lugano, Switzerland) on alternate days starting from day 7 and maintained every other day till the $10^{\text {th }}$ day of cycle, then dose monitored according to size of follicle.

Intramuscular injection of 5000 IU of highly purified hCG (Choriomon, IBSA, Lugano, Switzerland) was given. Timed coitus is asked within the following 24-40 hrs. Endometrial thickness was measured at the greatest diameter perpendicular to the midsagittal plane in the fundal region and serum estradiol on day of hCG. Serum progesterone was measured one week after ultrasonographic confirmed ovulation or one week from last examination in non-responders, where folliculometry continued for two weeks from the beginning of treatment. Serum B-subunit hCG was measured 2 weeks after triggering to diagnose pregnancy. Ultrasound examination was performed 5 weeks post triggering to detect the presence of fetal cardiac pulsations and to exclude any extrauterine pregnancy.

Ultrasound examination was done for all patients in the studied group using a SonoAce X4 machine (Meddison, Korea) with transvaginal probe (4-8 $\mathrm{MHz}$ frequency). Basal examination (on cycle day 3 ) was done prior to any medication to exclude the presence of any retention cyst $>2 \mathrm{~cm}$. During folliculometry evaluation of number, size of ovarian follicles in each ovary, endometrial thickness, frequency of visit was tailored according to size of follicle till dominance was confirmed (dominance means presence of follicle $>14 \mathrm{~mm}$ ) or excluded 2 weeks after the end of the treatment. Only one complete treatment cycle was offered to each woman of both groups.

The outcome measures included occurrence of pregnancy, ovulation rate, the number of mature follicles, serum E2 ( $\mathrm{pg} / \mathrm{ml})$, serum $\mathrm{P}(\mathrm{ng} / \mathrm{mL})$ and endometrial thickness $(\mathrm{mm})$ in addition to rate of miscarriage.

\section{RESULTS}

As shown in diagram 1, two hundred and twenty (220) anovulatory CC resistant PCOS couples were recruited in this study. Five cases were excluded before starting; three of them do not meet inclusion criteria, two with mild male factor, one with only one tube patent, one do not come back and one was delayed due to large retention cyst on basal US. The treatment regimen was given for two hundred fifteen (215) patients who were randomly divided into group A (107 patients) and group B (108 patients) for only one cycle. But, the results were carried out on two hundred and twelve patients due to dropping of three cases; two from group B (do not take gonadotropin injection due to its cost) and one from group A (the husband was not available, so she does not come back). Only one hundred and six cases in each group started treatment. There was no significant statistical difference between both groups $\mathrm{A}$ and $\mathrm{B}$, regarding their demographic criteria including age, BMI, duration and type of infertility, clinical (menstrual pattern) and laboratory variables (TSH, PRL, $\mathrm{LH})$ as shown in table (1).

In table (2), there was higher ovulation rate (about $85.8 \%$ ) in Letrozole $+\mathrm{hmG}$ group than in the other group (85 patients out of 106 ovulated) with about $80.2 \%$ in treatment regimen with Letrozole and CC. But, there was statistical significant difference between both groups regarding stimulation characters varying from total number of mature follicles (diameter $\geq 18 \mathrm{~mm}$ ) $2.1 \pm 1.01$ in combined Letrozole and $\mathrm{CC}$ group $\mathrm{A}$ which was greater than in group B $(1.66 \pm 0.98)$ with $P$ - value $=0.009$. With stimulation, days were longer in group B (18.2 \pm 2.5$)$ versus $16.2 \pm 2.1$ in group A. Mean total E2 on the day of hCG administration was significantly higher among patients in the Clomiphene-Letrozole group as compared with that in the other group $(650 \pm 190.3$ and $540 \pm 180.5$, respectively). Also, on day 21 , serum progesterone was higher in group A $(11 \pm 1.1)$ than in group B $(9.5 \pm 0.9)$. But, there was no statistical significant difference in mean endometrial thickness in both groups A and B $(9.6 \pm 1.7$ versus $9.8 \pm 1.5 \mathrm{~mm}$ ) with $P$ - value $=0.41$ Table (2). 


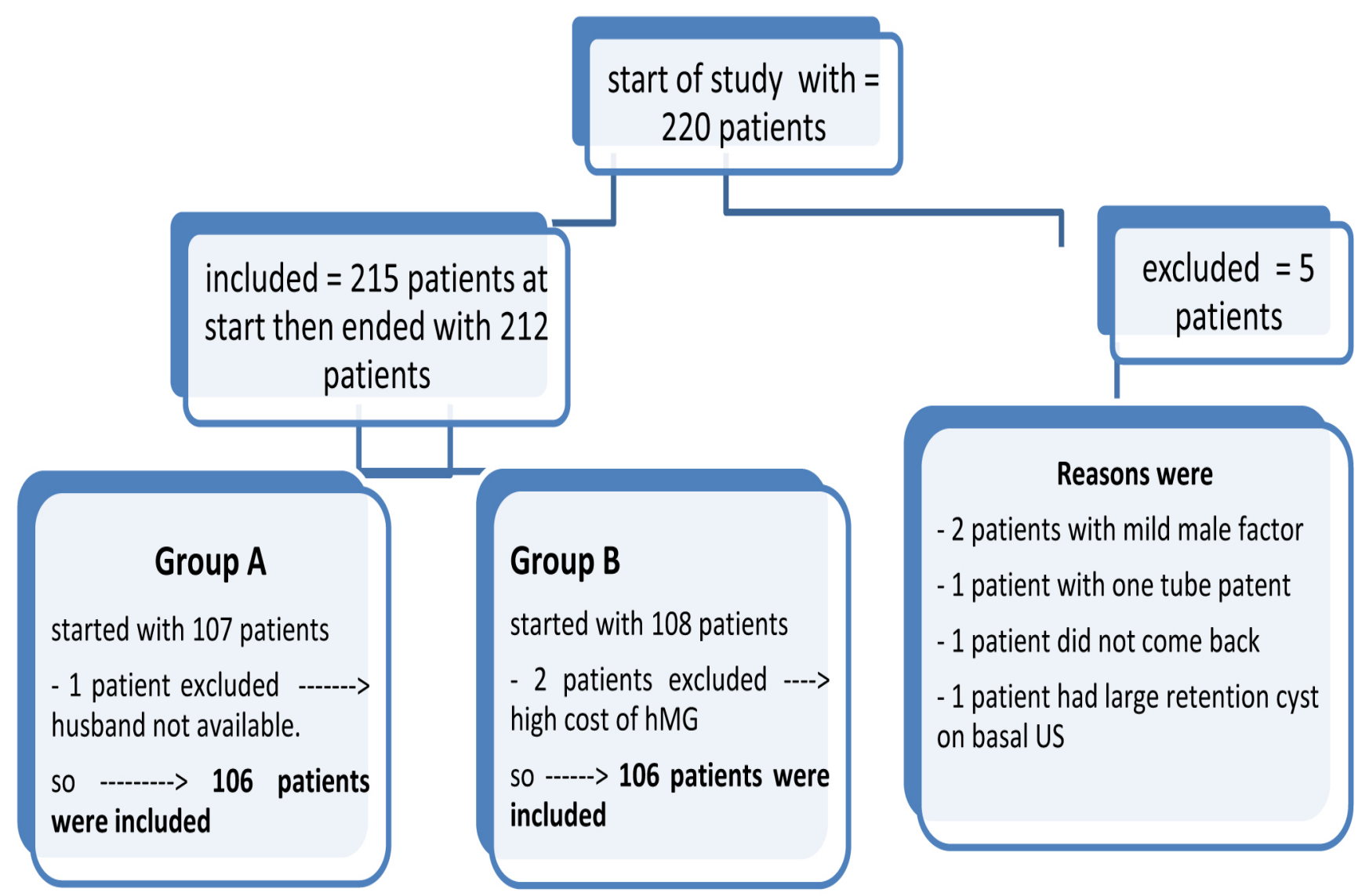

Diagram 1: Study design

The pregnancy rate did not show any statistical significant difference in both groups $(\mathrm{P}$ value $=0.45) ; 32$ patients (30.1\%) in group A compared with 27 patients (25.4\%) in group B. Four cases were aborted in group A and three cases in group B. Four cases had twin pregnancy in group A compared with three cases only in group B. Also, there was no significant difference between them regarding ovarian hyperstimulation; two cases with combined clomid in Letrozole group and one case only in Letrozole and hMG group as shown in Table (3).

Table 1: Demographic characters of studied group

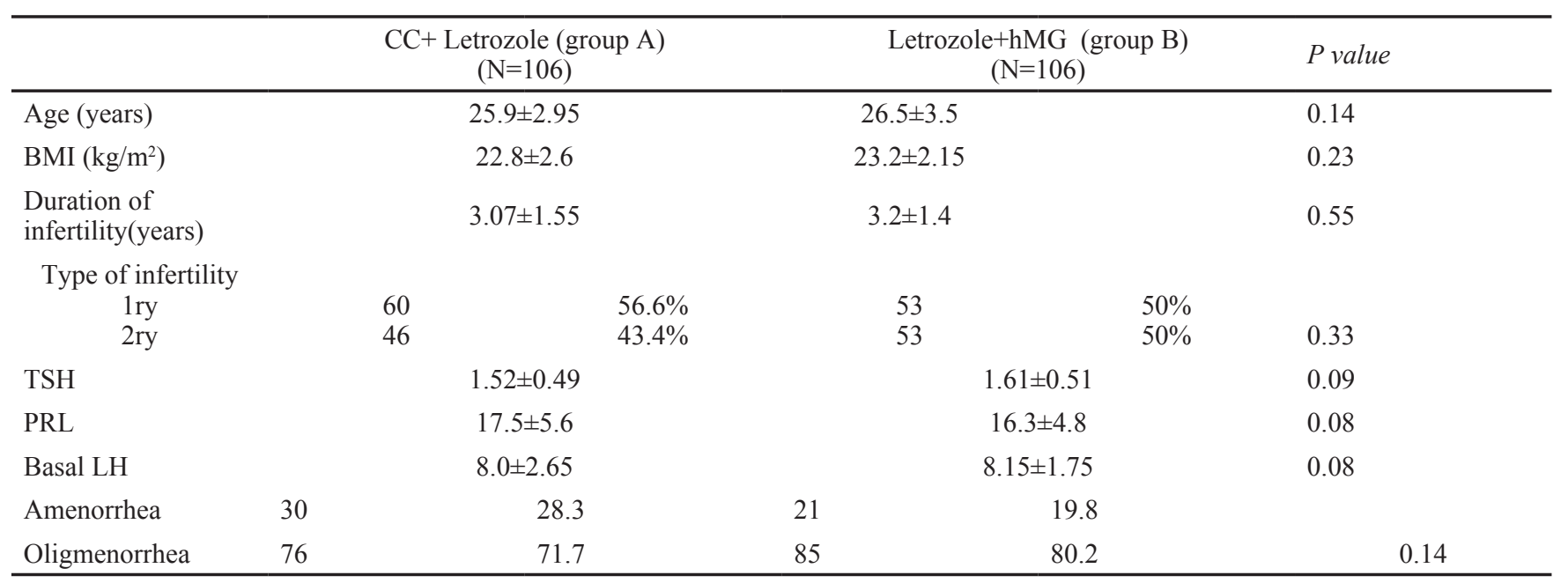

None of the variable was statistically significant different $(P$ value $>0.05)$ 
Table 2: Stimulation characters of studied group

\begin{tabular}{|c|c|c|c|c|c|}
\hline & \multicolumn{2}{|c|}{$\begin{array}{c}\mathrm{CC}+\text { Letrozole } \\
(\mathrm{N}=106)\end{array}$} & \multicolumn{2}{|c|}{$\begin{array}{l}\text { Letrozole+HMG } \\
\qquad(\mathrm{N}=106)\end{array}$} & $P$ \\
\hline No of follicle $\geq 18 \mathrm{~mm}$ & \multicolumn{2}{|c|}{$2.1 \pm 1.01$} & \multicolumn{2}{|c|}{$1.66 \pm 0.98$} & $0.009 *$ \\
\hline Cycle completed & 85 & $80.2 \%$ & 91 & $85.8 \%$ & 0.65 \\
\hline Stimulation days & \multicolumn{2}{|c|}{$16.2 \pm 2.1$} & \multicolumn{2}{|c|}{$18.2 \pm 2.5$} & $0.00 * *$ \\
\hline Endometrial thickness (mm) & \multicolumn{2}{|c|}{$9.6 \pm 1.7$} & \multicolumn{2}{|c|}{$9.8 \pm 1.5$} & 0.41 \\
\hline Serum E2 on hCG day (pg/ml) & \multicolumn{2}{|c|}{$650 \pm 190.3$} & \multicolumn{2}{|c|}{$540 \pm 180.5$} & $0.00 * *$ \\
\hline Serum prog (ng/ml) & \multicolumn{2}{|c|}{$11 \pm 1.1$} & \multicolumn{2}{|c|}{$9.5 \pm 0.9$} & $0.00 * *$ \\
\hline Monif cycle & 74 & $69.8 \%$ & 85 & $80.2 \%$ & 0.42 \\
\hline
\end{tabular}

Significance considered with $p$ value $<0.05 * *$.

Table 3: Outcome measures

\begin{tabular}{|c|c|c|c|c|c|}
\hline \multirow[b]{2}{*}{ Pregnancy rate } & \multicolumn{2}{|c|}{$\begin{array}{c}\mathrm{CC}+\text { Letrozole } \\
(\mathrm{N}=106)\end{array}$} & \multicolumn{2}{|c|}{$\begin{array}{l}\text { Letrozole }+\mathrm{hMG} \\
\qquad(\mathrm{N}=106)\end{array}$} & \multirow{2}{*}{$\begin{array}{c}\mathrm{P} \text { value } \\
0.45\end{array}$} \\
\hline & 32 & $30.1 \%$ & 27 & $25.4 \%$ & \\
\hline Abortion & 4 & $15.6 \%$ & 3 & $11.1 \%$ & 0.54 \\
\hline Multiple & 4 & $15.6 \%$ & 3 & $11.1 \%$ & 0,54 \\
\hline OHSS & 2 & $1.88 \%$ & 1 & $0.94 \%$ & 0.57 \\
\hline Ectopic & 0 & $0.0 \%$ & 0 & $0.0 \%$ & ---- \\
\hline
\end{tabular}

No statistical significant difference in all variables but with higher pregnancy rate in CC + Letrozole

\section{DISCUSSION}

Ovulatory disorders are considered the major cause of infertility, about $75 \%$ of chronic anovulation is PCOSrelated. Induction of ovulation is the proper treatment strategy for PCOS related infertility ${ }^{[16]}$.Clomiphene is still the first-line therapy for induction of ovulation in PCOS with ovulation rate $73 \%-80 \%{ }^{[4]}$. Failure to cause ovulation for three cycles is known as clomiphene citrate resistance, but failure to get pregnant in spite of good ovulation within 6-12 months is known as clomiphene citrate failure ${ }^{[4]}$. In addition, CC has low pregnancy rate (40\%) ; in spite of relatively higher ovulation rate that may be attributed to its antiestrogenic effect on endometrial development and cervical mucus production ${ }^{[17]}$.

In the last few years, different studies approved that aromatase inhibitors (Letrozole) is a good, effective and safe treatment modality in infertility and ovulation induction in $\mathrm{PCOS}^{[18]}$. Moreover, use of Letrozole has been recommended for patients who ovulate with clomiphene but have a thin endometrium ${ }^{[4]}$. Owing to its effectiveness, lower adverse effects and low cost, Letrozole can be used instead of gonadotropins in patients resistant to clomiphene. Moreover, Letrozole induces mono follicular development; so, the risk of multiple pregnancies is reduced. In addition, it is rapidly eliminated from the body; thus, lower risk of fetal anomaly ${ }^{[19]}$. In a cohort study, no significant difference was observed in the frequency of fetal anomaly with Letrozole $(2.4 \%)$ more with clomiphene $(3 \%)^{[20]}$.

Gonadotropins are also another treatment modality for PCOS patients resistant to clomiphene but its usage carries some disadvantage including serial hormonal measurements and sonographic monitoring with serious adverse effects including ovarian hyperstimulation syndrome, multiple pregnancies and high cost. Laparoscopic ovarian drilling also is an effective treatment in those CC-resistant patients who do not respond also to gonadotropins, or accept the cost and adverse effects of these drugs, but its usage is still a matter of controversy due to its temporary effects, risk of adhesions, and detrimental drawbacks on ovarian reserve ${ }^{[4]}$. 
It was found that resistance to clomiphene occurs mostly with high body mass index, amenorrhea, severe hyperandrogenemia and insulin-resistance ${ }^{[19]}$. Also, Letrozole alone can induce ovulation in $62 \%$ with pregnancy occurred in $14.7 \%$ in patients with improper response to clomiphene or CC resistant ${ }^{[11]}$. Combination of both drugs in those patients resistant to $\mathrm{CC}$ can potentiate each other with proper ovulation response in comparison with Letrozole and low dose hmG. In this study, there was higher ovulation rate about $85.8 \%$ in Letrozole + hMG group than in the other group (combined Letrozole + CC) $80.2 \%$, but this increase was not statistically significant; with pregnancy rate $30.1 \%$ and $25.4 \%$, respectively, in both groups. These results were convenient with the study done by Masomeh et al. $(2013)^{[15]}$ on one hundred infertile couples with PCOS wife resistant to clomiphene and Letrozole alone; they were treated (each one alone) for three cycles.

Fourteen patients became pregnant in the first cycle, 15 patients in the second cycle and 13 patients in the third cycle. In general, 257 cycles of combination therapy with clomiphene and Letrozole were done, in which 213 cycles resulted in the formation of a dominant follicle $(82.9 \%)$ with pregnancy rate $42 \%$.

Also, Rasekhjahromi Atharn et al. on 2015 found that combination of Clomid and Letrozole for induction of ovulation in PCOS patients has good ovulation rate $20 \%{ }^{[21]}$. Also, Abu Hashim et al. reported that $30 \%$ of their PCOS patients were resistant to clomiphene, but Letrozole induced an ovulation rate in those CC-resistant patients $(64.9 \%)$ with pregnancy rate of $14.7 \%{ }^{[11]}$.

Our study showed that there was no significant difference regarding abortion $15.6 \%$ for group $\mathrm{A}$ and $11.1 \%$ for group B, respectively. But Masomeh et al. (2013) reported that there was $23.8 \%$ abortion rate with combined $\mathrm{CC}$ and Letrozole therapy ${ }^{[15]}$. Also, Rasekhjahromi Athar et al. (2015) reported miscarriage rate of about $2.4 \%$ with CC plus Letrozole in PCOS infertile females ${ }^{[21]}$. Speroff and Fritz reported that the frequency of abortion following gonadotropins in their clomiphene-resistant patients was $20 \%-25 \%$, which is more than that in the general population (15\%). They also explained this by older age and number of obese subjects in their study population ${ }^{[4]}$.

Finally, from our study, we found that combination of $\mathrm{CC}$ and Letrozole can be effective in inducing ovulation in about $80.2 \%$ of CC-resistant patients with pregnancy rate of about $32 \%$ with no statistically significant difference from Letrozole combined with low dose hMG where ovulation rate is $85.8 \%$ with a pregnancy rate of about $27 \%$, so we had the same results but with low cost. More studies are needed to understand the effectiveness of the combination of drugs on a larger population of patients. The combination may also be used as first-line therapy to induce ovulation in severe cases of PCOS in order to save time and expense.

\section{CONFLICT OF INTEREST}

There are no conflict of IInterest.

\section{REFERENCES}

1. Teede H, Deeks A, Moran L. Polycystic ovary syndrome: a complex conditions with psychological, reproductive and metabolic manifestations that impact on health across the lifespan. BMC Med. 2010; 8:41.

2. Guzick D. Polycystic ovary syndrome. Symptomatology, pathophysiology and epidemiology. Am J Obstet Gynecol 1998; 179: 89-93.

3. Mitwally MF, Casper RF. Use of an aromatase inhibitor for induction of ovulation in patients with an inadequate response to clomiphene citrate. Fertil Steril. 2001; 75: 305-9.

4. Fritz M, Speroff L. Clinical Gyneocology endocrinology and infertility. $8^{\text {th }}$ ed. PhiladelphiaL. Lippincott Williams and Wilkins. 2011: 1305-13.

5. Blank SK, McCartney CR, Marshall JC. The origins and sequelae of abnormal neuroendocrine function in polycystic ovary syndrome.Hum Reprod Update 2006; 12(4):351-61.

6. Franks S, Adams J, Mason H, Polson D. Ovulatory disorders in women with polycystic ovary syndrome. Clin Obstet Gynecol, 1985; 12: 605-32.

7. Shelly W, Draper MW, Krishnan V, Wong M, Jaffe RB. Selective estrogen receptor modulators: an update on recent clinical findings. Obstet Gynecol Surv 2008; 63(3): 163-81.

8. Homburg R. Clomiphene citrate- end of an era? Mini review. Hum Reprod 2005; 20: 2043-51.

9. Al-Omari WR, Sulaiman WR, Al-Hadithi N. Comparison of two aromatase inhibitors in women with clomiphene-resistant polycystic ovary syndrome. Int J Gynaecol Obstet. 2004; 85(3): 289-91.

10. Requena A, Herrero J, Landeras J, Navarro E, Neyro JL,Salvador C, Tur R, et al. Use of letrozole in assisted reproduction: a systematic review and meta-analysis. Hum Reprod 2008; 14(6): 571-82.

11. Abu Hashim H, Shokeir T, Badawy A. Letrozole versus combined metformin and clomiphene citrate for ovulation induction in clomipheneresistant women with polycystic ovary syndrome: a randomized controlled trial. Fertil Steril. 2009; 94(4): 1405-9 
12. Pritts EA. Letrozole for ovulation induction and controlled ovarian hyperstimulation. Curr Opin Obstet Gynecol. 2010; 22(4): 189-94.

13. The Rotterdam ESHRE/ASRM-Sponsored PCOS Consensus Workshop Group. Revised 2003 consensus on diagnostic criteria and long-term health risks related to polycystic ovary syndrome. Fertil Steril, 2004; 19:25-81.

14. Wenyan Xi, Shankun Liu, Hui Mao, Yongkang Yang, Xiang Xue, Xiaoning Lu. Use of letrozole and clomiphene citrate combined with gonadotropins in clomiphene-resistant infertile women with polycystic ovary syndrome: a prospective study. Drug Design, Development and Therapy 2015; 9 : 6001-8

15. Masomeh h, Meisam DE, Nazila K, Nahideh Sa, Seyed N, Mohammad Gh. Combined letrozole and clomiphene versus letrozole and clomiphene alone in infertile patients with polycystic ovary syndrome, Drug combined Design, Development and Therapy 2013;8:1427-31.

16. Jirege PR, Patill RS. Comparison of endocrine and ultrasound profiles during ovulation induction with clomiphene citrate and letrozole in ovulatory volunteer women. Fertil Steril. 2010; 93(1): 174-183.
17. Gonen Y, Casper RF. Sonographic determination of a possible adverse effect of clomiphene citrate on endometrial growth. Hum Reprod. 1990; 5: 670-4.

18. Badawy A, Shokeir T, Allam AF, Abdolhady H. Pregnancy outcome after ovulation induction with aromastase inhibitors or clomiphene citrate in unexplained infertility. Acta Obstet Gynecol Scand. 2009; 88(2): 187-91.

19. Abu Hashim H, Shokeir T, Badawy A. Letrozole versus combined metformin and clomiphene citrate for ovulation induction in clomipheneresistant women with polycystic ovary syndrome: a randomized controlled trial. Fertil Steril. 2009; 94(4):1405-9.

20. Tulandi T, Martin J, Al-Fadhli R, et al. Congenital malformations among 911 newborns conceived after infertility treatment with letrozole or clomiphene citrate. Fertil Steril. 2006; 85(6):1761-5.

21. Rasekhjahromi Athar, Maalhagh Mehrnoosh, Hosseinpoor Masoumeh, Farhang Hooshmand and Alavi Fatemeh. A Clomiphene Citrate and Letrozol Varsus Tamoxifen and Letrozole as an Infertility Treatment in Women with Polycystic Ovary Syndrome. Pakistan Journal of Biological Sciences, 2015;18 (6): 300-3 